\title{
Level Crossing Rate in Terms of the Characteristic Function: A New Approach for Calculating the Fading Rate in Diversity Systems
}

\author{
Ali Abdi', Member, IEEE, and Mostafa Kaveh², Fellow, IEEE \\ ${ }^{l}$ Dept. of Elec. and Comp. Eng., New Jersey Institute of Technology, Newark, NJ 07102 \\ ${ }^{2}$ Dept. of Elec. and Comp. Eng., University of Minnesota, Minneapolis, MN 55455 \\ Emails: ali.abdi@njit.edu kaveh@ece.umn.edu
}

Abstract _ The level crossing rate (LCR) of a random process conveys useful information about the underlying process, and is of interest in diverse engineering fields. In wireless communications, it is related to the system characteristics such as handoff, outage probability, fading rate, average duration of fades, velocity (or maximum Doppler shift) of the mobile, and the effect of diversity on fading. The LCR formula was originally derived by Rice in terms of the joint probability density function (PDF) of the underlying process and its time derivative. In this paper we express the LCR in terms of the joint characteristic function (CF). This new formula is useful for many cases where the joint $\mathrm{CF}$ is simpler to derive than the associated joint PDF. As an application and for a DS-CDMA system, the fading rate at the output of a RAKE receiver with either maximal ratio combiner or postdetection equal gain combiner, operating over a frequency-selective fading channel with different path statistics, is easily calculated using the new CF-based LCR formula.

Keywords: Characteristic function, DS-CDMA systems, Fading channels, Level-crossing problems, RAKE receivers.

This work was supported in part by the National Science Foundation, under the Wireless Initiative Program, Grant \#9979443. 


\section{INTRODUCTION}

Wireless communications systems are subject to the fading, and diversity-based techniques are powerful tools for mitigating the impact of fading [1]. The basic idea of all diversity methods is to combine several independent copies of the signal at the receiver. Application of multiple antennas at the receiver is an efficient and common diversity reception. Utilization of the RAKE receiver for wideband systems (which are impaired by frequency-selective fading) is another type of diversity reception [1]. In the real world, the paths over which the signal has propagated are not independent and identically distributed (i.i.d.) [2] [3] [4] [5]. So, the replicas of the transmitted signal at the receiver have different fading distributions, and are mutually correlated. Direct sequence-code division multiple access (DS-CDMA) systems operating over frequency-selective fading channels, are typical examples where we encounter non-i.i.d. fading among the paths.

Fading rate in a diversity system, defined as the level crossing rate (LCR) of the total instantaneous signal-to-noise ratio (SNR) per symbol at the output of the diversity combiner, is a useful measure for evaluating the dynamic performance of that diversity system. The common approach for calculating the LCR of a random process is to employ Rice's formula, which expresses the LCR in terms of the joint probability density function (PDF) of the process and its derivative. However, for diversity systems operating over non-i.i.d. fading channels, it is very hard, if not impossible, to derive an expression for the joint PDF of instantaneous SNR and its derivative at the output of the diversity combiner. On the other hand, for cases of interest such as Rayleigh and Rice fading channels, the associated joint characteristic function (CF) can be derived in closed form. Therefore, Rice's LCR formula for a random process should be rewritten in terms of the joint CF of the process and its derivative. In what follows, such a formula has been derived for an arbitrary random process and its utility has been demonstrated by calculating the fading rate at the output of a DSCDMA RAKE receiver with either maximal ratio combining (MRC) or postdetection equal gain combining (EGC), operating over a Rice-Rayleigh frequency-selective fading channel.

\section{THE LCR FORMULA IN TERMS OF THE CHARACTERISTIC FUNCTION}

For a given level $\ell$, and according to the celebrated Rice's formula, the LCR of a stationary random process $Y(t)$ is given by [6]:

$E\left[N_{\ell}\{Y(t)\}\right]=\int_{-\infty}^{\infty}\left|y^{\prime}\right| p_{Y Y^{\prime}}\left(\ell, y^{\prime}\right) d y^{\prime}$,

where $E$ is the mathematical expectation, $N_{\ell}\{Y(t)\}$ represents the number of times, per unit time, 
where $Y(t)=\ell$, prime denotes differentiation with respect to time $t$, and $p_{Y Y^{\prime}}\left(y, y^{\prime}\right)$ is the joint PDF of the random variables $Y=Y\left(t_{0}\right)$ and $Y^{\prime}=Y^{\prime}\left(t_{0}\right)$, with $t_{0}$ as an arbitrary instant of time. Now we want to express $E\left[N_{\ell}\{Y(t)\}\right]$ in terms of $\Phi_{Y Y^{\prime}}\left(\omega_{1}, \omega_{2}\right)=E\left[\exp \left(j \omega_{1} Y+j \omega_{2} Y^{\prime}\right)\right]$, the joint CF of $Y$ and $Y^{\prime}$, rather than $p_{Y Y^{\prime}}\left(y, y^{\prime}\right)$ (note that $\left.j^{2}=-1\right)$. Using the generalized Parseval's theorem for the right-hand side of (1), noting that $\left|y^{\prime}\right|=y^{\prime} \operatorname{sgn}\left(y^{\prime}\right)$ with $\operatorname{sgn}($.$) as the signum function, we obtain:$

$$
E\left[N_{\ell}\{Y(t)\}\right]=\frac{1}{2 \pi} \int_{-\infty}^{\infty} \mathfrak{I}\left[\operatorname{sgn}\left(y^{\prime}\right)\right]\left\{\mathfrak{I}\left[y^{\prime} p_{Y Y^{\prime}}\left(\ell, y^{\prime}\right)\right]\right\}^{*} d \omega_{2}=\frac{1}{2 \pi} \int_{-\infty}^{\infty} \mathfrak{I}\left[y^{\prime} \operatorname{sgn}\left(y^{\prime}\right)\right]\left\{\mathfrak{I}\left[p_{Y Y^{\prime}}\left(\ell, y^{\prime}\right)\right]\right\}^{*} d \omega_{2},
$$

where $\mathfrak{I}[$.$] is the Fourier transform and *$ is the complex conjugate operator. Based on:

$$
\mathfrak{I}\left[\operatorname{sgn}\left(y^{\prime}\right)\right]=2 /\left(j \omega_{2}\right), \quad \mathfrak{I}\left[p_{Y Y^{\prime}}\left(\ell, y^{\prime}\right)\right]=(2 \pi)^{-1} \int_{-\infty}^{\infty} \Phi_{Y Y^{\prime}}\left(-\omega_{1},-\omega_{2}\right) e^{j \omega_{1} \ell} d \omega_{1}
$$

and the properties of the Fourier transform we finally obtain:

$$
E\left[N_{\ell}\{Y(t)\}\right]=\frac{-1}{2 \pi^{2}} \int_{-\infty}^{\infty} \int_{-\infty}^{\infty} \frac{1}{\omega_{2}} \frac{d \Phi_{Y Y^{\prime}}\left(\omega_{1}, \omega_{2}\right)}{d \omega_{2}} e^{-j \omega_{1} \ell} d \omega_{1} d \omega_{2}=\frac{-1}{2 \pi^{2}} \int_{-\infty}^{\infty} \int_{-\infty}^{\infty} \frac{\Phi_{Y Y^{\prime}}\left(\omega_{1}, \omega_{2}\right)-\Phi_{Y}\left(\omega_{1}\right)}{\omega_{2}{ }^{2}} e^{-j \omega_{1} \ell} d \omega_{1} d \omega_{2} .
$$

For cases where the derivation of $\Phi_{Y Y^{\prime}}\left(\omega_{1}, \omega_{2}\right)$ is easier than $p_{Y Y^{\prime}}\left(y, y^{\prime}\right)$, (2) plays an important role. An example of such a case is reported in [7], where an exact and simple solution for the expected number of maxima of the envelope of a spherically invariant random process is derived. Based on that simple solution, which could not be obtained using Rice's LCR formula in (1), a new velocity estimator for cellular systems, which is robust against the variations of SNR and channel characteristics, is proposed in [8]. In the sequel, we will see how equation (2) allows us to solve an LCR problem which seems to be intractable using Rice's formula given in (1).

\section{FADING RATE AT THE OUTPUT OF MRC AND PostdeteCtion EGC}

Assume that the diversity combiner has $Q$ branches ( $Q$ fingers in the RAKE receiver). In the presence of additive white Gaussian noise, which is independent of fading, the instantaneous SNR per symbol of the $q$ th branch is given by $\gamma_{q}(t)=R_{q}{ }^{2}(t) /\left(N_{0} B\right)$. In this formula $R_{q}(t)$ is the signal envelope of the $q$ th branch with the average power $\Omega_{q}=E\left[R_{q}{ }^{2}(t)\right], N_{0}$ is the one-sided power spectral density of the noise, and $B$ is the bandwidth of the receive filter in each branch. Clearly, the average SNR per symbol of the $q$ th branch can be written as $\bar{\gamma}_{q}=\Omega_{q} /\left(N_{0} B\right)$. The total instantaneous SNR per symbol at the output of both MRC and postdetection EGC is given by [1]:

$\gamma(t)=\sum_{q=1}^{Q} \gamma_{q}(t)$

The total average SNR per symbol at the output of the combiners can be written as 
$\bar{\gamma}=\sum_{q=1}^{Q} \bar{\gamma}_{q}=\sum_{q=1}^{Q} \Omega_{q} /\left(N_{0} B\right)$. From now on, let us assume $N_{0} B=1$, to simplify the notation without loss of generality. We define $F R_{\gamma_{h}}$, the fading rate with respect to the given threshold $\gamma_{t h}$ at the output of the diversity combiner, as the $\gamma_{t h}$-crossing rate of $\gamma(t)$, i.e. $F R_{\gamma_{t h}}=E\left[N_{\gamma_{t h}}\{\gamma(t)\}\right]$.

In this paper we assume that separate resolvable propagation paths are independent. However, the paths can be distributed according to a common fading distribution with different parameter values [9], or even different families of fading distributions [10]. Note that such a general setting is not just of theoretical interest, because wideband measurements of frequency-selective fading channels have shown non-identically distributed paths [2] [3] [4] [5]. In this paper, we consider a diversity combiner, with the first $Q_{1}$ branches having a Rice-distributed envelope and the second $Q_{2}=Q-Q_{1}$ branches having a Rayleigh-distributed envelope. This model corresponds to a typical frequency-selective fading channel in a mixed urban-suburban region [3].

In order to calculate $E\left[N_{\gamma_{t h}}\{\gamma(t)\}\right]$ using (2), we first derive an expression for $\Phi_{\gamma_{q} \gamma_{q}^{\prime}}\left(\omega_{1}, \omega_{2}\right)$, assuming $R_{q}(t)=\sqrt{\gamma_{q}(t)}$ has a Rice distribution [1]:

$$
p_{R_{q}}\left(r_{q}\right)=\frac{r_{q}}{b_{q, 0}} \exp \left(-\frac{r_{q}^{2}+a_{q}^{2}}{2 b_{q, 0}}\right) I_{0}\left(\frac{a_{q} r_{q}}{b_{q, 0}}\right), \quad r_{q} \geq 0,
$$

where $I_{0}($.$) is the modified Bessel function of order zero, and a_{q}$ and $b_{q, 0}$ are nonnegative numbers described in the sequel. Based on the inphase and quadrature representation for $R_{q}(t)$ we have $R_{q}(t)=\sqrt{U_{q}^{2}(t)+V_{q}^{2}(t)}$, where $U_{q}(t)$ is a Gaussian process with mean $a_{q}$ and variance $b_{q, 0}$, while $V_{q}(t)$ is a zero-mean Gaussian process with the same variance $b_{q, 0}$, independent of $U_{q}(t)$. Therefore:

$$
\Phi_{\gamma_{q} \gamma_{q}^{\prime}}\left(\omega_{1}, \omega_{2}\right)=E\left[\exp \left(j \omega_{1} \gamma_{q}+j \omega_{2} \gamma_{q}^{\prime}\right)\right]=E\left[\exp \left\{j \omega_{1}\left(U_{q}^{2}+V_{q}^{2}\right)+j 2 \omega_{2}\left(U_{q} U_{q}^{\prime}+V_{q} V_{q}^{\prime}\right)\right\}\right]
$$

Let us define the following vectors and matrices:

$$
\begin{aligned}
& \mathbf{W}_{q}=\left[\begin{array}{llll}
U_{q} & V_{q}^{\prime} & V_{q} & U_{q}^{\prime}
\end{array}\right]^{T}, \quad \boldsymbol{\mu}_{q}=\left[\begin{array}{llll}
a_{q} & 0 & 0 & 0
\end{array}\right]^{T}, \\
& \boldsymbol{\Sigma}_{q}=\left[\begin{array}{cccc}
b_{q, 0} & b_{q, 1} & 0 & 0 \\
b_{q, 1} & b_{q, 2} & 0 & 0 \\
0 & 0 & b_{q, 0} & -b_{q, 1} \\
0 & 0 & -b_{q, 1} & b_{q, 2}
\end{array}\right], \quad \mathbf{D}=\left[\begin{array}{cccc}
\omega_{1} & 0 & 0 & \omega_{2} \\
0 & 0 & \omega_{2} & 0 \\
0 & \omega_{2} & \omega_{1} & 0 \\
\omega_{2} & 0 & 0 & 0
\end{array}\right],
\end{aligned}
$$

with $T$ as the transpose operator. Since differentiation is a linear operation, the processes $U_{q}^{\prime}(t)$ and $V_{q}^{\prime}(t)$ are also Gaussian, with zero mean and the same variance $b_{q, 2}$. So, $\mathbf{W}_{q}$ is a Gaussian vector with the mean-vector and the covariance-matrix given in (6). Let us also define the lowpass equivalent complex envelope of the $q$ th branch as $\Re_{q}(t)=R_{q}(t) \exp \left[j \Theta_{q}(t)\right]$, where $\Theta_{q}(t)=\tan ^{-1}\left[V_{q}(t) / U_{q}(t)\right]$ is 
the signal phase of the $q$ th branch. We represent the autocovariance function of $\mathfrak{R}_{q}(t)$ by $\phi_{\Re_{q} \Re_{q}}(\tau)$, and the associated power spectrum by the Fourier transform $S_{\mathfrak{\Re}_{q} \Re_{q}}(f)=\mathfrak{I}\left[\phi_{\mathfrak{R}_{q} \Re_{q}}(\tau)\right]$. The parameter $b_{q, n}, n=0,1,2$ in (6) is the $n$th spectral moment of $\Re_{q}(t)$, defined by [6]:

$b_{q, n}=(2 \pi)^{n} \int_{-\infty}^{\infty} f^{n} S_{\Re_{q} \Re_{q}}(f) d f$.

Using the properties of the Fourier transform, it is easy to show that:

$$
b_{q, n}=j^{-n} d^{n} \phi_{\Re_{q} \Re_{q}}(\tau) /\left.d \tau^{n}\right|_{\tau=0} .
$$

Based on the definitions in (6), (5) can be written as:

$$
\Phi_{\gamma_{q} \gamma_{q}^{\prime}}\left(\omega_{1}, \omega_{2}\right)=E\left[\exp \left(j \mathbf{W}_{q}^{T} \mathbf{D} \mathbf{W}_{q}\right)\right]
$$

Since $\mathbf{W}_{q}$ in (6) is a Gaussian vector with the mean-vector $\boldsymbol{\mu}_{q}$ and the covariance-matrix $\boldsymbol{\Sigma}_{q}$, the scalar random variable $\mathbf{W}_{q}{ }^{T} \mathbf{D} \mathbf{W}_{q}$ is a quadratic form of Gaussian variables, and its characteristic function $E\left[\exp \left(j \omega \mathbf{W}_{q}{ }^{T} \mathbf{D} \mathbf{W}_{q}\right)\right]$ can be evaluated according to [11]:

$$
E\left[\exp \left(j \omega \mathbf{W}_{q}{ }^{T} \mathbf{D} \mathbf{W}_{q}\right)\right]=\frac{\exp \left(-\boldsymbol{\mu}_{q}{ }^{T} \boldsymbol{\Sigma}_{q}{ }^{-1}\left[\mathbf{I}-\left(\mathbf{I}-j 2 \omega \boldsymbol{\Sigma}_{q} \mathbf{D}\right)^{-1}\right] \boldsymbol{\mu}_{q} / 2\right)}{\sqrt{\operatorname{det}\left(\mathbf{I}-j 2 \omega \boldsymbol{\Sigma}_{q} \mathbf{D}\right)}},
$$

where $\mathbf{I}$ is the $4 \times 4$ unit matrix and $\operatorname{det}\left(\right.$.) denotes determinant. Now $\Phi_{\gamma_{q} \gamma_{q}^{\prime}}\left(\omega_{1}, \omega_{2}\right)$ in (8) can be easily derived by replacing $\omega$ in (9) with 1 . After some algebraic manipulations we obtain:

$$
\Phi_{\gamma_{q} \gamma_{q}^{\prime}}\left(\omega_{1}, \omega_{2}\right)=\frac{\exp \left(-\frac{a_{q}{ }^{2}\left(2 b_{q, 2} \omega_{2}{ }^{2}-j \omega_{1}\right)}{1+4\left(b_{q, 0} b_{q, 2}-b_{q, 1}{ }^{2}\right) \omega_{2}{ }^{2}-j 2 b_{q, 0} \omega_{1}}\right)}{1+4\left(b_{q, 0} b_{q, 2}-b_{q, 1}{ }^{2}\right) \omega_{2}{ }^{2}-j 2 b_{q, 0} \omega_{1}} .
$$

For a Rayleigh distribution, $a_{q}=0,(10)$ significantly simplifies to:

$$
\Phi_{\gamma_{q} \gamma_{q}^{\prime}}\left(\omega_{1}, \omega_{2}\right)=\left[1+4\left(b_{q, 0} b_{q, 2}-b_{q, 1}{ }^{2}\right) \omega_{2}^{2}-j 2 b_{q, 0} \omega_{1}\right]^{-1}
$$

Based on the independence of $\gamma_{q}(t) \mathrm{s}$ and the definition of $\gamma(t)$ in (3), we have:

$$
\Phi_{\gamma^{\prime}}\left(\omega_{1}, \omega_{2}\right)=\prod_{q=1}^{Q} \Phi_{\gamma_{q} \gamma_{q}^{\prime}}\left(\omega_{1}, \omega_{2}\right)
$$

Substitution of (11) into (2) gives the fading rate $E\left[N_{\gamma_{t h}}\{\gamma(t)\}\right]$.

Now suppose the diversity combiner is mounted on a mobile receiver. If the scattering over the $q$ th path to the mobile receiver is nonisotropic, then $b_{q, n}$ for all $n$ can be different from zero [12]. However, for isotropic scattering we have $\phi_{\Re_{q} \Re_{q}}(\tau)=b_{q, 0} J_{0}\left(2 \pi f_{m} \tau\right)$, where $J_{0}($.$) is the Bessel function of order$ zero and $f_{m}$ is the maximum Doppler frequency [1] $\left(f_{m}\right.$ is related to the speed of the mobile $v$ according to $v=f_{m} \lambda_{c}$, with $\lambda_{c}$ as the wavelength of the carrier). So, the spectral moments are: 
$b_{q, 1}=0, \quad b_{q, 2}=2 \pi^{2} f_{m}^{2} b_{q, 0}$.

As a result, (10) reduces to:

$\Phi_{\gamma_{q} \gamma_{q}^{\prime}}\left(\omega_{1}, \omega_{2}\right)=\frac{\exp \left(-\frac{a_{q}{ }^{2}\left(4 \pi^{2} f_{m}{ }^{2} b_{q, 0} \omega_{2}{ }^{2}-j \omega_{1}\right)}{1+8 \pi^{2} f_{m}{ }^{2} b_{q, 0}{ }^{2} \omega_{2}{ }^{2}-j 2 b_{q, 0} \omega_{1}}\right)}{1+8 \pi^{2} f_{m}{ }^{2} b_{q, 0}{ }^{2} \omega_{2}{ }^{2}-j 2 b_{q, 0} \omega_{1}}$.

It is common to express $a_{q}$ and $b_{q, 0}$ in terms of Rice factor $K_{q}$ and the average power $\Omega_{q}$, defined by $K_{q}=a_{q}{ }^{2} /\left(2 b_{q, 0}\right) \quad$ and $\quad \Omega_{q}=a_{q}{ }^{2}+2 b_{q, 0} \quad[1]$. By substituting $a_{q}{ }^{2}=K_{q} \Omega_{q} /\left(K_{q}+1\right) \quad$ and $2 b_{q, 0}=\Omega_{q} /\left(K_{q}+1\right)$ into (13) we obtain:

$\Phi_{\gamma_{q} \gamma_{q}^{\prime}}\left(\omega_{1}, \omega_{2}\right)=\frac{\left(K_{q}+1\right)^{2} \exp \left(-\frac{\Omega_{q} K_{q}\left[2 \pi^{2} f_{m}{ }^{2} \Omega_{q} \omega_{2}{ }^{2}-j\left(K_{q}+1\right) \omega_{1}\right]}{\left(K_{q}+1\right)^{2}+2 \pi^{2} f_{m}{ }^{2} \Omega_{q}^{2} \omega_{2}{ }^{2}-j \Omega_{q}\left(K_{q}+1\right) \omega_{1}}\right)}{\left(K_{q}+1\right)^{2}+2 \pi^{2} f_{m}{ }^{2} \Omega_{q}^{2} \omega_{2}{ }^{2}-j \Omega_{q}\left(K_{q}+1\right) \omega_{1}}$.

For the Rayleigh distribution, $K_{q}=0,(14)$ drastically simplifies to:

$\Phi_{\gamma_{q} \gamma_{q}^{\prime}}\left(\omega_{1}, \omega_{2}\right)=\left(1+2 \pi^{2} f_{m}^{2} \Omega_{q}^{2} \omega_{2}^{2}-j \Omega_{q} \omega_{1}\right)^{-1}$.

To see the difference between the fading rates of combiners with non-i.i.d. and i.i.d. branches, we consider two numerical examples: a non-i.i.d. case and an i.i.d. case. In the non-i.i.d. scenario we have $Q=3$ and $Q_{1}=1$. This means Rice-distributed envelope in the first branch and Rayleigh-distributed envelopes in the second and third branches. Therefore, $K_{2}=K_{3}=0$. Among the proposed models for the power delay profile we consider the common equally-spaced exponential profile [13]:

$\Omega_{q}=\Omega_{1} e^{-\rho(q-1)}, \quad \rho \geq 0, \quad q=1,2, \ldots, Q$,

where the parameter $\rho$ reflects the rate at which the average power decays. By substituting (14) into (11) and then into (2), $F R_{\gamma_{t h}}$ for the non-i.i.d. case is plotted in Fig. 1, using the Mathematica(C) software for these parameter values: $K_{1}=10(10 \mathrm{~dB}), \Omega_{1}=7(8.45 \mathrm{~dB}), \rho=0.2$, and $f_{m}=5 \mathrm{~Hz}$, which corresponds to the mobile speed $v=1.5 \mathrm{~m} / \mathrm{s}$, if the carrier frequency $f_{c}$ is $1 \mathrm{GHz}$. Notice that $\Omega^{(\text {non-iid })}=\sum_{q=1}^{Q} \Omega_{q}=[1+\exp (-\rho)+\exp (-2 \rho)] \Omega_{1} \approx 17.43(12.41 \mathrm{~dB})$. For the i.i.d. scenario $Q=3$ and $Q_{1}=0$. This implies Rayleigh-distributed envelope for all the branches. Hence, $K_{1}=K_{2}=K_{3}=0$. We also assume $\rho=0$, so $\Omega_{1}=\Omega_{2}=\Omega_{3}$. For this case we take $\Omega_{1}=5.81(7.64 \mathrm{~dB})$, which yields $\Omega^{(i i d)}=\sum_{q=1}^{Q} \Omega_{q}=3 \Omega_{1}=17.43(12.41 \mathrm{~dB})=\Omega^{(\text {non-iid })}$. The corresponding $F R_{\gamma_{t h}}$ for the i.i.d. case is also plotted in Fig. 1. Visual comparison of these two plots exhibits the significant difference between the non-i.i.d. and i.i.d. cases, about which a system designer should always be aware.

For the case of i.i.d. branches described above, $\gamma(t)$ in (3) is a chi-square process with 6 degrees 
of freedom, where the variance of all the six underlying Gaussian components is $\Omega_{1} / 2$. Based on the LCR of a chi process derived in [14], $F R_{\gamma_{t h}}$ for the above i.i.d.-branches example can be written as:

$$
F R_{\gamma_{t h}}=\sqrt{2 \pi} f_{m}\left(\gamma_{t h} / \Omega_{1}\right)^{5 / 2} \exp \left(-\gamma_{t h} / \Omega_{1}\right)
$$

The numerical results obtained by our approach comply very accurately with (16) as a special case.

\section{CONCLUSION}

In this paper we have introduced a characteristic function (CF)-based approach for calculating the level crossing rate (LCR) of a random process, as a new alternative to the traditional probability density function (PDF)-based method, first proposed by Rice. As an application, we have considered a DS-CDMA system, and calculated the fading rate at the output of a RAKE receiver, operating over a frequency-selective fading channel with different path statistics. Such a problem defies even a

numerically tractable solution using Rice's LCR formula [15]. As the last word, the LCR of an equal gain combiner is calculated in [16], where the joint CF is first derived, then its Fourier transform, the joint PDF, is computed and plugged into Rice's LCR expression. However, as we have shown here, the LCR can be directly expressed in terms of the CF and there is no need to calculate the associated PDF.

\section{REFERENCES}

[1] G. L. Stuber, Principles of Mobile Communication. Boston, MA: Kluwer, 1996.

[2] P. C. Fannin and A. Molina, "Analysis of mobile radio channel sounding measurements in inner city Dublin at 1.808 Ghz," IEE Proc. Commun., vol. 143, pp. 311-316, 1996.

[3] K. A. Stewart, G. P. Labedz, and K. Sohrabi, "Wideband channel measurements at $900 \mathrm{MHz,"} \mathrm{in} \mathrm{Proc.}$ IEEE Vehic. Technol. Conf., Chicago, IL, 1995, pp. 236-240.

[4] W. R. Braun and U. Dersch, "A physical mobile radio channel model," IEEE Trans. Vehic. Technol., vol. 40, pp. 472-482, 1991.

[5] H. Suzuki, “A statistical model for urban radio propagation,” IEEE Trans. Commun., vol. 25, pp. 673-680, 1977.

[6] S. O. Rice, "Mathematical analysis of random noise," reprinted in Selected Papers on Noise and Stochastic Processes. N. Wax, Ed., New York: Dover, 1954, pp. 133-294.

[7] A. Abdi and S. Nader-Esfahani, "Expected number of maxima in the envelope of a spherically invariant random process," revised version submitted to IEEE Trans. Inform. Theory, Jul. 2001.

[8] A. Abdi and M. Kaveh, "A new velocity estimator for cellular systems based on higher order crossings," in Proc. Asilomar Conf. Signals, Systems, Computers, Pacific Grove, CA, 1998, pp. 1423-1427.

[9] G. P. Efthymoglou, V. A. Aalo, and H. Helmken, "Performance analysis of coherent DS-CDMA systems in a 
Nakagami fading channel with arbitrary parameters," IEEE Trans. Vehic. Technol., vol. 46, pp. 289-297, 1997.

[10] R. D. J. van Nee, H. S. Misser, and R. Prasad, "Direct-sequence spread spectrum in a shadowed Rician fading land-mobile satellite channel," IEEE J. Select. Areas Commun., vol. 10, pp. 350-357, 1992.

[11] G. L. Turin, "The characteristic function of Hermitian quadratic forms in complex normal variables," Biometrika, vol. 47, pp. 199-201, 1960.

[12] A. Abdi, H. Allen Barger, and M. Kaveh, "A parametric model for the distribution of the angle of arrival and the associated correlation function and power spectrum at the mobile station," accepted for publication in IEEE Trans. Vehic. Technol..

[13] M. K. Simon and M. S. Alouini, Digital Communication over Fading Channels: A Unified Approach to Performance Analysis. New York: Wiley, 2000.

[14] R. A. Silverman, "The fluctuation rate of the chi process," IRE Trans. Inform. Theory, vol. 4, pp. 30-34, 1958.

[15] A. M. Hasofer, "The upcrossing rate of a class of stochastic processes," in Studies in Probability and Statistics. E. J. Williams, Ed., New York: North-Holland, 1974, pp. 153-159.

[16] W. C. Y. Lee, Mobile Communications Engineering. New York: McGraw-Hill, 1982.

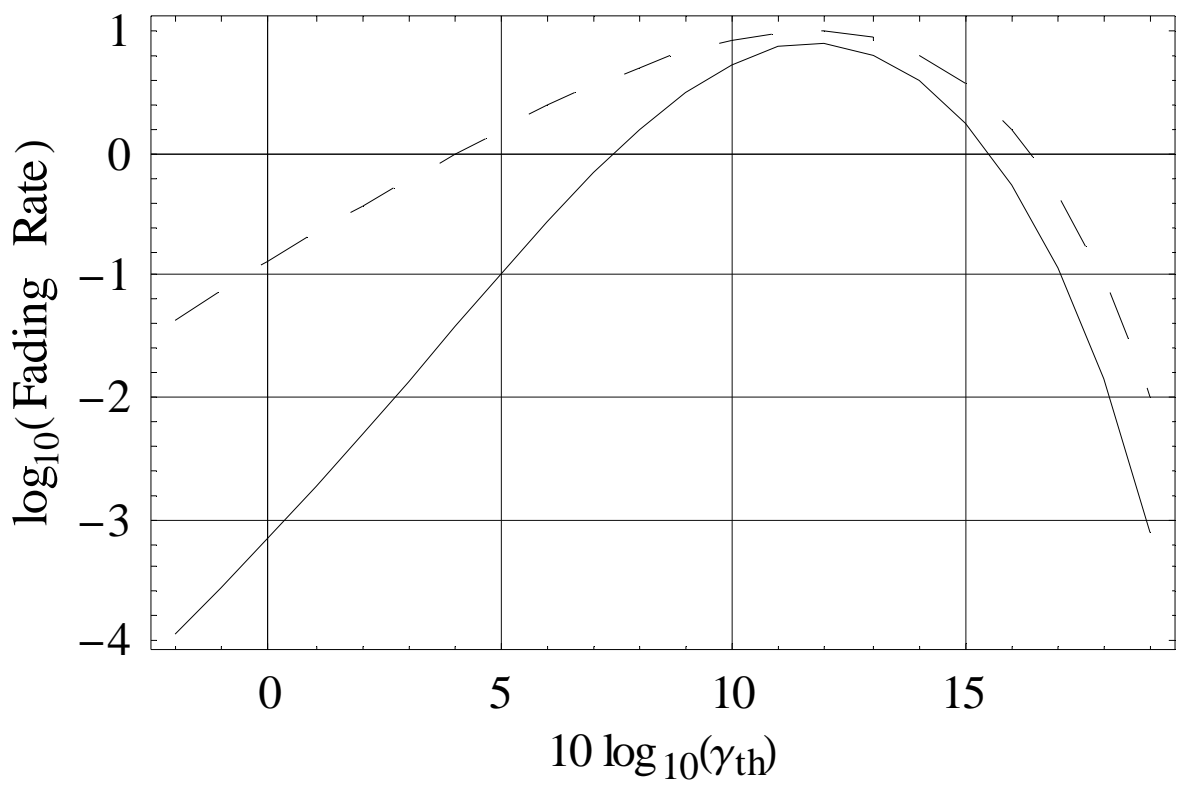

Fig. 1. Fading rate versus the SNR threshold $\gamma_{t h}(\mathrm{~dB})$ at the output of a three-branch MRC or postdetection EGC RAKE receiver.

non-i.i.d. branches, Rice distribution for the first branch and Rayleigh distributions with different powers for the second and the third branches

- - - - i.i.d. branches with Rayleigh distribution 